\title{
GESTÃO DE PROJETOS: GESTÃO DE ESCOPO E STAKEHOLDERS NA IMPLANTAÇÃO DE UM PROJETO DE SOFTWARE EM UM AMBIENTE SEM CONTEXTO DE PROJETOS
}

\author{
SCOPE AND STAKEHOLDER MANAGEMENT IN THE \\ IMPLEMENTATION OF A SOFTWARE PROJECT IN AN \\ ENVIRONMENT WITHOUT PROJECT CONTEXT
}

\author{
Juliano Idogawa ${ }^{1}$ \\ Silvia Regina Veronese Correia $^{2}$ \\ Flavio Santino Bizarrias ${ }^{3}$
}

\begin{abstract}
Resumo: Este relato técnico discute a importância da aplicação de ferramentas de gerenciamento de projetos, com foco em gestão de escopo e stakeholders. O método utilizado foi estudo de caso e a unidade de análise foi um projeto de implementação de software de um fornecedor multinacional para concessão de descontos em academias de ginástica numa empresa de transportes aéreos no Brasil. Os dados foram coletados por meio de análise documental e entrevista com o gerente do projeto da companhia. A conclusão da análise dos dados deste estudo permitiu o entendimento dos problemas ocorridos no gerenciamento do projeto e como as dificuldades das áreas que tratam de gestão de escopo e stakeholders encontradas durante a gestão do projeto culminaram no atraso do projeto. A intervenção executada no modelo de assessoria permitiu realizar um mapa de problemas versus lições aprendidas. A contribuição deste trabalho é consolidada pela elaboração de uma proposta de gestão de escopo e stakeholders utilizando as boas práticas de gerenciamento de projetos em contextos que não sejam de projetos.
\end{abstract}

Palavras-chave: Gerenciamento de escopo. Gerenciamento de stakeholders. Gerenciamento de projetos

\begin{abstract}
This technical report discusses the importance of applying project management tools focused on scope and stakeholder management. The method used was a case study and the unit of analysis was a software implementation project of a multinational supplier for the granting of discounts in fitness centers in an air transport company in Brazil. Data were collected through document analysis and interviews with the project manager at the company. The conclusion of the data analysis of this study allowed us to understand the problems that occurred in project management and how the difficulties encountered during project management involving the areas that deal with scope management and stakeholders culminated in the project delay. The intervention performed in the advisory model allowed us to draw a map of problems versus lessons learned. The contribution of this work is consolidated by the preparation of a scope and stakeholder management proposal using good project management practices in non-project contexts.
\end{abstract}

Keywords: Scope management. Stakeholder management. Project management

${ }^{1}$ Mestrando em Administração - Gestão de Projetos, Universidade Nove de Julho - Uninove. São Paulo, SP - Brasil. juliano.idogawa@outlook.com

${ }^{2}$ Mestranda em Administração - Gestão de Projetos, Universidade Nove de Julho - Uninove. São Paulo, SP - Brasil. silviaveronezi@ gmail.com

Dי3rofessor stricto sensu, Pesquisador , e Consultor empresarial, Universidade Nove de Julho - Uninove, São Paulo, SP - Brasil. flavioxsp@ hotmail.com

\section{Cite como}

American Psychological Association (APA)

Idogawa, J., Correia, S. R. V., \& Bizarrias, F. S. (2020, jul./dez.). Gestão de projetos: gestão de escopo e stakeholders na implantação de um projeto de software em um ambiente sem contexto de projetos. Revista Inovação, Projetos e Tecnologias, São Paulo, 8(2), 168-180. https://doi.org/10.5585/iptec.v8i2.17456. 


\section{Introdução}

O objetivo deste relato técnico é discutir a importância da adoção de boas práticas no gerenciamento de projetos com foco na gestão adequada no detalhamento de escopo e mapeamento dos stakeholders para o sucesso de projetos. Como objeto de estudo (Biancolino, Kniess, Maccari, \& Rabechini, 2012), foi utilizado um projeto de implantação de software com a finalidade de gerar descontos entre empresas parceiras. $\mathrm{O}$ projeto foi realizado em uma empresa de transportes aéreos que conta com aproximadamente 21.000 funcionários no Brasil.

O caso relatado evidencia as competências relevantes do gerente de projeto e a necessidade de aplicar as ferramentas de gestão de projetos. Kerzner (2015) cita que uma das habilidades necessárias ao gerente de projetos é a sua competência para coordenar as ações técnicas necessárias ao projeto. No caso reportado, a área de Segurança da Informação da empresa, responsável por validar quesitos de segurança de produtos e serviços de software, não foi incluída entre os stakeholders. A falta da gestão de stakeholders no início do projeto resultou no congelamento dos trabalhos por conta da ausência de requisitos necessários e não mapeados (gerenciamento do escopo).

Segundo Carvalho e Rabechini (2019), é fundamental identificar os principais grupos de stakeholders do projeto, mapear suas influências e gerenciar suas expectativas ao longo de todo o ciclo de vida dos projetos. É por meio do correto envolvimento dos stakeholders que a declaração de escopo pode ser elaborada com as principais entregas, tornando claro o limite do projeto e o trabalho necessário para executá-lo (PMI, 2017).

Neste relato técnico foi utilizada a abordagem qualitativa com o método de estudo de caso, cujo objetivo foi analisar de forma empírica os problemas de um projeto de implantação de software e propor um direcionamento baseado em lições aprendidas, ancorado nas melhores práticas da gestão de projetos para evitar que o mesmo cenário encontrado no objeto de estudo se repita em projetos futuros.

O procedimento metodológico foi composto por análise de documentos, entrevista, triangulação dos dados coletados com a teoria sobre gerenciamento de projetos e elaboração de uma proposta de gestão com base na literatura. Verificou-se a ausência da gestão de stakeholders e de escopo como a principal fonte dos problemas encontrados no caso relatado. A contribuição prática deste trabalho foi propor uma sistematização na gestão de projetos de acordo com as boas práticas consolidadas em gestão de projetos com foco em escopo e stakeholders em ambientes sem contexto ou tradição em projetos. 
Seguem nas próximas seções deste relato técnico: referencial teórico, procedimento metodológico e intervenção, contexto do projeto, resultados obtidos e análise, considerações finais e conclusão.

\section{Referencial teórico}

\subsection{Competências técnicas na gestão de projetos}

O conceito de competência, segundo Carvalho e Rabechini (2019), contempla as habilidades, conhecimentos e atitudes necessárias para se atingir resultados. $\mathrm{Na}$ visão de Lampel (2001), as competências dividem-se em técnica, avaliadora, empreendedora e relacional. As competências técnicas envolvem o trabalho técnico de diagnóstico, habilidades específicas, uso de ferramentas e trabalho de sistematização. Nesse sentido, de acordo com Carvalho e Rabechini (2019), a adoção de técnicas e ferramentas de gerenciamento de projetos é fundamental e tem sido uma preocupação das empresas.

Ainda segundo Carvalho e Rabechini (2019), em termos de gerenciamento de projetos, a utilização de técnicas e ferramentas como boas práticas em gestão visa garantir o sucesso dos projetos. De acordo com Patah e Carvalho (2012), é possível constatar a influência positiva e significativa no sucesso de projetos por meio de do uso de ferramentas de gestão. Nesse sentido, seguindo as práticas consolidadas pelo PMI (2017) em gerenciamento de projetos, as ferramentas devem ser utilizadas para controlar os projetos seguindo um conjunto de áreas de conhecimento: integração, escopo, tempo, custos, qualidade, recursos, comunicação, riscos, aquisições e partes interessadas. Nessa diretriz, a ferramenta para controle de escopo é a estrutura analítica de projetos (EAP) e, para controle de stakeholders, a matriz de stakeholders, que avalia o nível de engajamento dentro do projeto. Ambas devem estar presentes no plano geral do projeto (Carvalho \& Rabechini, 2019).

É preciso salientar que diversos autores (Carvalho \& Rabechini, 2019; Drejer, 2001; Drejer \& Riis, 1999; Lampel, 2001; Loufrani-Fedida \& Missonier, 2015), defendem que as competências para a gestão de projetos complementam os fatores necessários e até mesmo essenciais para o sucesso de projetos.

\subsection{Gerenciamento de stakeholders}

Segundo Freeman (1984), stakeholders são indivíduos ou grupos que podem afetar ou serem afetados pelos objetivos da empresa. Esse conceito é ratificado pela definição do PMI (2017), que estabelece que stakeholders podem ser pessoas, grupos ou organizações com poder 
de impactar ou serem impactadas por uma decisão, atividade ou resultado do projeto ao longo de todo o ciclo de vida do projeto, de forma positiva ou negativa.

Os stakeholders podem ser representados por partes como empresas, entidades de classe ou órgãos governamentais, que podem ter uma ligação formal ou informal com o projeto. A influência exercida pelos stakeholders em diferentes momentos pode gerar modificações nos resultados esperados do próprio projeto em diversas proporções (Carvalho \& Rabechini, 2019). Assim, algumas entregas não planejadas no escopo inicial podem ser necessárias para o atendimento das necessidades dos interessados (Kerzner, 2015).

Conforme Carvalho e Rabechini (2019), os stakeholders podem impactar ou serem impactados por decisões, atividades ou resultado do projeto e têm expectativas relacionadas ao seu resultado. Essas partes podem se manifestar a qualquer momento do projeto e gerar impactos de diferentes naturezas, a exemplo do caso que é tratado neste relato. Ainda segundo os autores, quanto mais complexo é o projeto, mais difícil é mapear seus respectivos stakeholders e priorizá-los por meio de dos impactos que podem causar. Dessa forma, é muito importante aplicar as práticas de gestão que contemplam a administração dessas partes e, principalmente, as inferências que elas possuem nos projetos.

Em seus estudos, Freeman (2010) apresentou uma ferramenta para a gestão dos stakeholders que identifica as dimensões de poder e interesse de cada um deles, criando uma matriz com essas informações. O poder está relacionado com a autoridade do stakeholder e quanto suas decisões podem interferir no projeto, enquanto o interesse está vinculado ao engajamento dentro do projeto. Essa matriz permite uma visão simplificada dos stakeholders dentro da estrutura do projeto.

Figura 1 - Matriz de interesse e poder

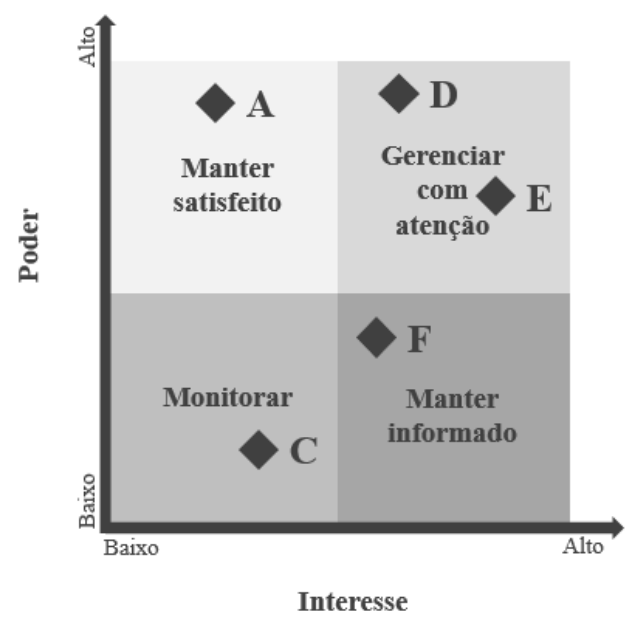

Fonte: Carvalho e Rabechini (2019). 


\subsection{Gerenciamento de escopo}

Segundo Carvalho e Rabechini (2019), escopo é o trabalho a ser realizado dentro do contexto do projeto e pode estar ligado ao produto do projeto ou ao próprio projeto; seu gerenciamento inclui que sejam executadas somente as atividades necessárias para seu sucesso, evidenciando a necessidade de planejamento e controle.

Seguindo as boas práticas na gestão de projetos, para que o planejamento seja realizado adequadamente é necessário que todas as informações estejam disponíveis no início do projeto, dentre elas estão as especificações do projeto e o termo de abertura do projeto (Kerzner, 2015). Inclusa no processo de gestão de escopo está a coleta dos requisitos gerados pelos stakeholders, que devem ser documentados e gerenciados (Carvalho \& Rabechini, 2019). Para Kerzner (2015), o escopo é a soma de todas as entregas que compõem o projeto, que podem ser desmembradas por meio da EAP. Por meio da EAP, poderão ser definidas de forma explicita as responsabilidades dos stakeholders (Carvalho \& Rabechini, 2019).

Figura 2 - Estrutura analítica do projeto

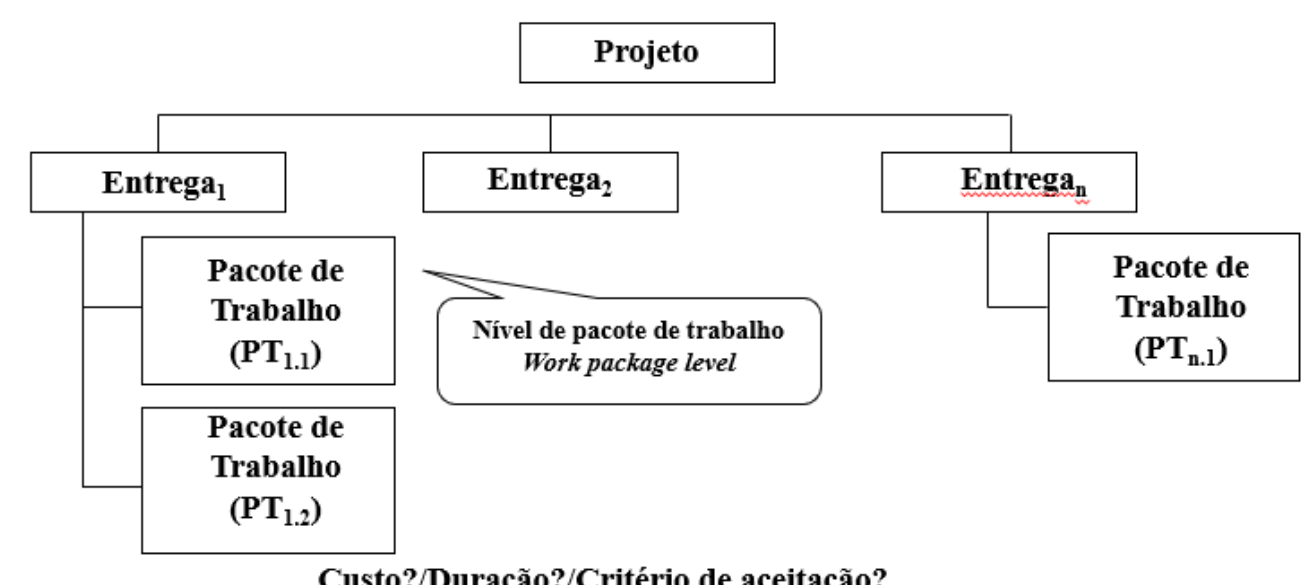

Custo?/Duração?/Critério de aceitação?

Fonte: Carvalho \& Rabechini (2019).

\section{Métodos da produção técnica e intervenção}

Neste trabalho foi utilizado o método estudo de caso: um tipo de pesquisa com investigação empírica que estuda um fenômeno dentro do contexto de vida real. Om método se divide em definição e planejamento, coleta de dados e análise e análise e conclusão (Yin, 2003). 
Figura 3 - Método de estudo de caso

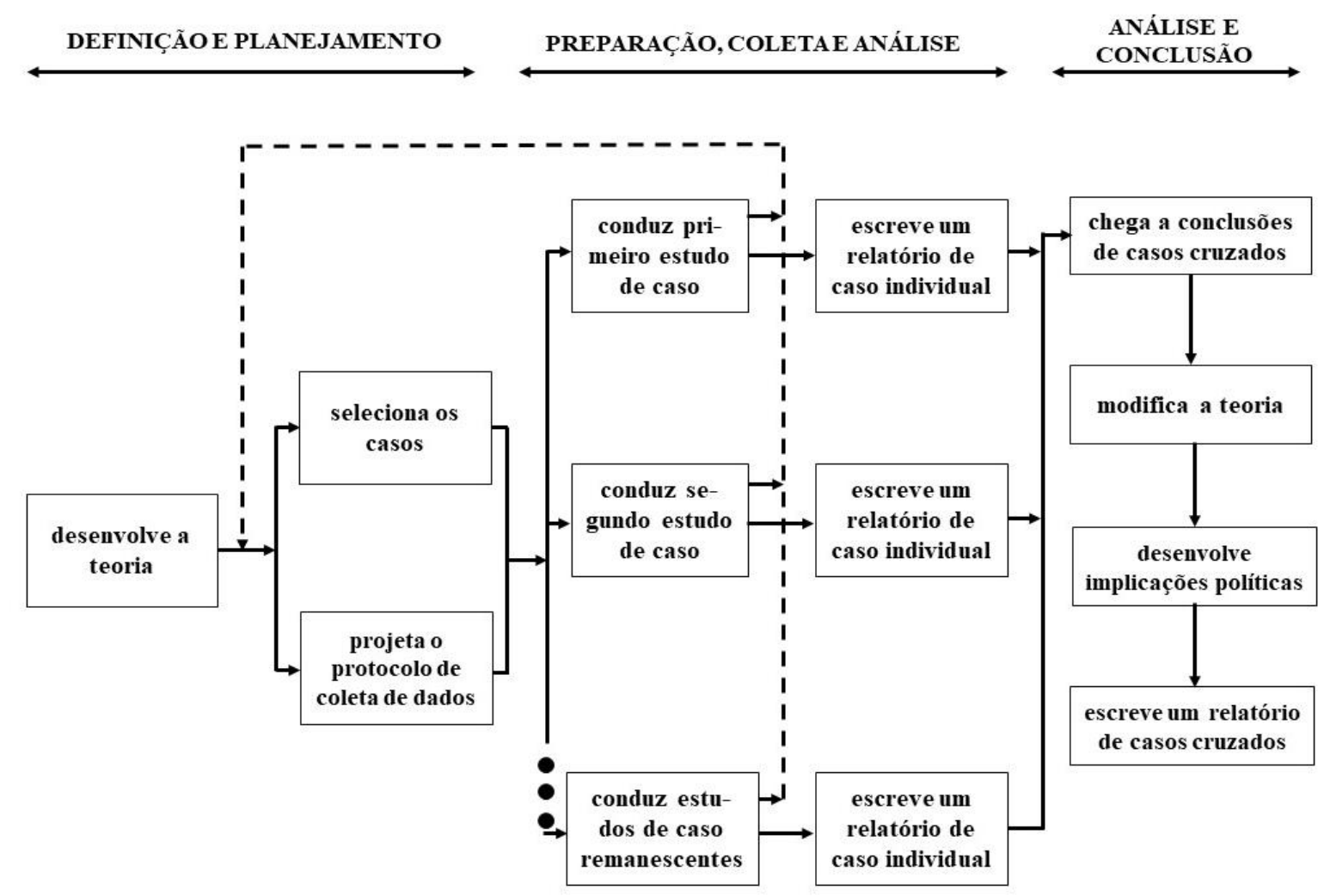

Fonte: Yin (2003).

Este método permitiu interação e proporcionou um melhor entendimento e detalhamento da situação problema. Um dos autores é funcionário da organização e atuou como participante observador.

Etapa 1 - Análise e levantamento dos dados

Para a pesquisa foram solicitados e analisados diversos documentos classificados como essenciais para a gestão de projetos, como plano de negócio (business case), termo de abertura do projeto (project charter), cronograma do projeto, plano de gerenciamento do projeto, plano de comunicação, plano de riscos, EAP e da reunião de abertura (Kick Off) do projeto.

Etapa 2 - Realização da entrevista

Ainda como fonte de dados foi realizada uma entrevista com o gerente da área de Qualidade de Vida (QV), pessoa que atuou como gerente do projeto para melhor compreender as características do projeto e processos de gestão com foco em escopo e stakeholders, com base nos autores Carvalho e Rabechini, (2019). 


\section{Etapa 3 - Intervenção}

O tipo de intervenção utilizada neste relato foi o de assessoria, que representa um “trabalho de acompanhamento e avaliação de um processo ou método existente (ou não) na organização" (Biancolino et al., 2012).

A primeira ação foi a criação de uma apresentação ao gerente da área QV com a finalidade de expor o levantamento dos problemas encontrados na triangulação de dados (documentos e entrevista) e obter seu apoio para execução das ações identificadas como necessárias para uma melhor gestão dos próximos projetos. Nessa apresentação foram utilizados alguns indicadores de desempenho de projetos baseados no PMI (2017). Em seguida, foi apresentada a forma como os projetos são controlados pelo Project Management Office (PMO), da área de tecnologia da empresa e como seu modelo de controle pode ser estendido para outros tipos de projeto. Foi apresentado, ainda, um cálculo de custo indireto do tempo de atraso do projeto para salientar a importância de um controle mais rígido da tríade escopo, custo e prazo.

Finalmente, foi entregue um relatório explicando como mapear escopo, como elaborar uma EAP com todas as entregas que o projeto deve conter, como controlar os requisitos a partir da matriz de rastreabilidade de requisitos e como monitorar o controle de mudanças de escopo. Nesse mesmo relatório foi incluída uma matriz de poder e interesse salientando a importância de mapear todos os envolvidos no projeto e como eles podem impactar o resultado do projeto. Foi demonstrada, ainda, a influência do gerente de projeto na identificação dos stakeholders e como ele tem um papel influenciador no posicionamento deles.

Outro ponto destacado foi a apresentação do PMBOK (PMI, 2017) ao gestor para que ele pudesse, a partir de uma leitura específica, entender o funcionamento de todas as áreas de conhecimento que devem ser controladas por um projeto considerando uma visão clássica.

\section{Contexto do projeto ou situação problema}

Este relato foi realizado em uma empresa multinacional latino-americana com aproximadamente 21.000 funcionários no Brasil. O projeto foi idealizado e gerenciado pela área de Recursos Humanos (RH), mais especificamente pelo departamento de Qualidade de Vida (QV), como um benefício aos colaboradores da companhia. A área de RH foi contatada pelo fornecedor que gerencia o software de descontos para academias a fim de realizar uma 
parceria entre ambas e o RH demonstrou interesse em implantar esse benefício já utilizado por outras empresas.

$\mathrm{Na}$ apresentação da proposta pelo fornecedor foram discutidos o modelo de implementação da parceria, bem como a adoção de uma proposta de implementação sem custo de projeto para o $\mathrm{RH}$. Posteriormente, a área de $\mathrm{QV}$ realizou todos os procedimentos internos da companhia até a aprovação do projeto. Foram envolvidas as áreas jurídica e financeira da empresa, além do próprio RH. Em nenhum momento a área de TI foi acionada, mesmo se tratando da implantação de um projeto que tinha como produto a disponibilização de um software a ser utilizado por todos os funcionários da empresa no Brasil.

A institucionalização da gestão de projetos é abordada por Carvalho e Rabechini (2019) como uma das competências que a organização deve possuir. A responsabilidade quanto à gestão da implantação foi atribuída ao gerente da área de QV com o apoio do fornecedor do software. Notou-se que o projeto foi tratado como uma compra de produto e não como um projeto em si. O formato de contratação do projeto não utilizou nenhuma metodologia ou ferramenta formal de gerenciamento de projetos e não alocou nenhum gerente de projetos para gerenciar o projeto.

O gerente da área de QV, desconhecendo as ferramentas de gestão adequadas, não seguiu as boas práticas de projetos que começam com o termo de abertura do projeto (Project Charter), que dá sequência ao envolvimento das partes interessadas, início do levantamento de requisitos (escopo) e construção do plano de projeto elencando quais as fases e entregas do projeto e como realizar o controle sobre elas (Kerzner, 2015). A execução do projeto foi feita a partir da negociação entre o departamento jurídico da companhia e fornecedor e o envolvimento das partes interessadas ocorreu pontualmente, bem como o levantamento de cada requisito e desenvolvimento de cada item necessário para realizar a implantação do benefício. A tabela 1 relaciona os stakeholders mapeados e não mapeados durante a fase inicial do projeto.

Tabela 1 - Mapeamento de stakeholders

\begin{tabular}{llll} 
Envolvidos & Tipo & Não mapeados & Tipo \\
RH / QV & Depto. Interno & PMO & Depto. Interno \\
\hline Compras & Depto. Interno & TI & Depto. Interno \\
\hline Jurídico & Depto. Interno & Segurança da & Depto. Interno \\
& & Informação & \\
Financeiro & Depto. Interno & & \\
\hline Fornecedor & Terceiros & &
\end{tabular}

Fonte: Autores. 
O cronograma proposto inicialmente pelo gerente de QV para o projeto seguiu o prazo de 3 meses informado pelo fornecedor, levando em conta o tempo de assinatura de contrato entre as empresas e em nenhum momento outros requisitos foram considerados. A elaboração e execução de um cronograma de acordo com o planejamento é uma das habilidades do gerente de projetos relacionadas por Kerzner (2015). O gerente de projetos não elaborou um cronograma formal e nenhum outro documento além do plano de comunicação aos funcionários da companhia.

O ponto de maior relevância que levou o projeto ao atraso em relação ao prazo inicialmente proposto foi identificado durante a entrevista. Devido ao desconhecimento das regras da área de tecnologia da informação, principalmente no quesito segurança de dados, o gerente de QV foi surpreendido quando o fornecedor solicitou a liberação de acessos à rede interna de dados da companhia para realizar o acesso ao novo software. Na ocasião a área de tecnologia da informação (TI) foi envolvida e iniciou-se o questionamento do modelo de segurança de dados a ser utilizado nessa conexão e os impedimentos das regras de segurança. O questionamento sobre a legislação especifica sobre a proteção de dados pessoais (LGPDP) deu origem a uma negociação jurídica, não prevista inicialmente no contrato (utilizado como plano para o projeto). Essa negociação causou ao projeto o atraso de 2 meses, ou seja, um atraso geral de $66 \%$ na data final inicialmente acordada.

Para atender a Lei n ${ }^{\circ} 13.709$, de 14 de Agosto de 2018 (LGPDP), nenhum dado pessoal dos funcionários como CPF (cadastro de pessoa física) ou RG (carteira de identidade) seriam informados ao fornecedor, dados esperados pelo contrato inicial. A fim de manter o projeto sem custo interno, não foi desenvolvido nenhum mecanismo que permitisse aos funcionários autorizar a utilização desses dados. A solução foi enviar os dados de matrícula do empregado na companhia, sem referenciar quaisquer outros dados dos funcionários e colaboradores, permitindo que o projeto continuasse com custo apenas para o fornecedor, que concordou em alterar o modelo de conexão para a empresa aérea e concluir o projeto. Observando esse cenário, foi constatada a ausência de ferramentas de gestão de projetos, o que levou os autores deste relato a propor uma intervenção na área para melhorar o gerenciamento dos próximos projetos.

\section{Resultados obtidos e análise}

A partir da intervenção, foi possível realizar uma retrospectiva do projeto mapeando as lições aprendidas a partir de uma perspectiva que utilizasse as boas práticas de gerenciamento de projetos focando em gerenciamento de escopo e stakeholders. 
Utilizamos como referência em gerenciamento de projetos o modelo sugerido pelo PMI (2017), com o propósito de sistematizar um método de controle de gestão e, assim, colaborar com a melhoria da eficiência na condução dos projetos realizados pela área de QV. A intenção desse mapa foi evitar que os mesmos erros fossem cometidos em futuros projetos, além de criar uma base de conhecimento. 
Tabela 2 - Problemas do projeto e boas práticas

\section{Problema do projeto}

Ausência de formalização do projeto. $O$ projeto relatado foi tratado como uma compra de produto e não como um projeto mesmo tendo recursos, prazo e escopo teoricamente definidos.

\section{Ausência de stakeholders; \\ Falta de envolvimento de stakeholders. Responsabilidades pouco ou não definidas.}

Ausência de requisitos.

Mapeamento de escopo incompleto. Ausência de controle de requisitos. Não utilização de ferramentas de controle de entregas.

Pouca ou nenhuma gestão de mudança.

Estimativa de prazo pouco assertiva. Pouco ou nenhum controle de prazo.

\section{Boas práticas de gerenciamento de projeto}

Conforme Carvalho e Rabechini (2019), o projeto deve ser formalizado através da elaboração de um documento chamado Termo de abertura do projeto (Project charter). O documento autoriza o gerente de projetos a aplicar os recursos disponibilizados pela organização para o desenvolvimento do projeto.

Ainda segundo os autores, o termo de abertura contém elementos que estabelece as necessidades de utilização de recursos, premissas e restrições. Esse documento deve conter: título, objetivo, premissas, restrições, resultados esperados, escopo macro, organização dos interessados, principais riscos.

Os envolvidos direta ou indiretamente devem ser mapeados, pois eles têm influência e responsabilidades no projeto. Uma ferramenta para controlar os stakeholders é matriz de stakeholders que pode ser construída em tabela contendo os seguintes campos: importância do stakeholder para o projeto, suas responsabilidades, expectativas, grau de interesse e poder e o nível de engajamento dentro do projeto. (Carvalho \& Rabechini, 2019). Segundo o PMI (2017), o gerenciamento do escopo do projeto inclui os $\mathrm{p}$ rocessos necessários para assegurar que o projeto inclua todo o trabalho, e apenas o necessário, para que termine com sucesso. O escopo do produto (aquilo que descreve um produto, serviço ou resultado), escopo do projeto (aquilo que deve ser realizado para entregar produto, serviço ou resultado com características e funções especificadas), restrições, premissas, pacotes de entregas (EAP), critérios de aceitação. Para o levantamento do escopo podem ser utilizados opinião dos especialistas, análise de dados, reuniões, entre outros (PMI 2017). Como ferramentas para o levantamento de escopo podem ser utilizados: coleta de requisitos com entrevista, diagramas, protótipos, análise de documentos, brainstorm, benchmarking, diagrama de contexto, mapeamento mental, protótipos. Segundo o PMI (2017), a documentação dos requisitos descreve como os requisitos individuais atendem as necessidades de negócio do projeto. Os requisitos podem começar em um alto nível e tornarem-se progressivamente mais detalhados, conforme mais informações sobre estes são conhecidas. Depois de mapeados eles devem estar contidos numa matriz de rastreabilidade que deve conter nome do projeto, unidade de negócio, identificador do requisito, identificadores associados, objetivo do negócio, objetivo do projeto, código na EAP, item do produto, mudanças autorizadas (Carvalho \& Rabechini, 2019).

Qualquer mudança deve ser mapeada e autorizada para que não comprometa o projeto.

As solicitações de mudança devem conter nome do projeto, gerente do projeto, nome do patrocinador, data, número de controle da mudança, área solicitante, objetivo da mudança, justificativa e benefícios da mudança, descrição, impactos positivos e negativos, custo, recursos necessários, prazo, riscos, premissas, urgência, impacto no escopo, documentos de suporte, aprovações, datas de aprovação (Carvalho \& Rabechini, 2019).

$\mathrm{O}$ cronograma deve ser elaborado definindo as atividades numa linha do tempo de forma a entregar os pacotes descritos na EAP. O sequenciamento das atividades permite estabelecer as relações de dependência. As atividades devem ser estimadas levando em consideração os recursos necessários. Depois do concluída a primeira, é gerada uma linha base que servirá para controlar as entregas e comparar o planejamento versus o realizado do projeto (Carvalho \& Rabechini, 2019).

Fonte: Autores. 


\section{Considerações finais e conclusão}

Este relato técnico discute a relevância das boas práticas de gestão de projetos principalmente a gestão de stakeholders e escopo. Outras áreas de conhecimento na gestão de projetos como gestão de riscos, integração, comunicação, aquisições, qualidade, tempo, custos e recursos humanos são relevantes ao tema, mas não foram objetos deste estudo.

Este trabalho destaca a importância de se envolver todas as partes necessárias para compor o escopo adequado para um projeto, além da requerida experiência do gerente de projetos, tanto na utilização de um método formal que permita coordenar a implantação de um projeto, quanto controlá-lo de maneira adequada. Além disso, evidencia a necessidade do conhecimento técnico do gerente do projeto, considerado como competências essenciais por diversos autores (Carvalho \& Rabechini, 2019; Kerzner, 2015).

Por meio deste relato foi possível identificar os motivos que levaram o projeto ao atraso e, a partir de uma análise geral, propor um modelo de gestão a partir das melhores práticas de gerenciamento de projetos, focadas em gestão de escopo e stakeholders. Buscou-se, por meio das lições aprendidas, evitar que os mesmos erros sejam cometidos em futuros projetos. Novos trabalhos ou relatos de natureza técnica e ou profissional podem ser realizados quanto à aplicação de ferramentas de gestão de projetos, em empresas cujos departamentos tenham autonomia para aquisição direta e sem avaliação do escritório de projetos.

\section{Referências}

Biancolino, C. A., Kniess, C. T., Maccari, E. A., \& Rabechini, R., Jr. (2012). Protocolo para Elaboração de Relatos de Produção Técnica. Revista de Gestão e Projetos, 3(2), 294-307.

Carvalho, M. M., \& Rabechini, R., Jr. (2019). Fundamentos em gestão de projetos: construindo competências para gerenciar projetos. (5a ed.). São Paulo: Atlas.

Drejer, A. (2001). How can we define and understand competencies and their development? Technovation, 21(3), 135-146.

Drejer, A., \& Riis, J. O. (1999). Competence development and technology How learning and technology can be meaningfully integrated. 631-644.

Freeman, R. E. (1984). Strategic management: A stakeholder approach. Boston, MA: Pitman/Ballinger.

Freeman, R. E. (2010). Strategic management: A stakeholder approach. Cambridge University Press. 
Kerzner, H. (2015). Gerenciamento de Projetos: uma Abordagem Sistêmica Para Planejamento, Programação e Controle (1a ed.). São Paulo: Blucher.

Lampel, J. (2001). The core competencies of effective project execution: the challenge of diversity. International Journal of Project Management, 471-483.

Lei n ${ }^{\circ}$ 13.709, de 14 de Agosto de 2018. Lei Geral de Proteção de Dados Pessoais (LGPDP). Recuperado em 23 de agosto, 2019, de http://www.planalto.gov.br/ccivil_03/_ato20152018/2018/lei/L13709.htm

Loufrani-Fedida, S., \& Missonier, S. (2015). The project manager cannot be a hero anymore! Understanding critical competencies in project-based organizations from a multilevel approach. International Journal of Project Management, 33(6), 1220-1235.

Patah, L.A., \& Carvalho, M. M. (2012). Métodos de Gestão de Projetos e Sucesso dos Projetos: Um Estudo Quantitativo do Relacionamento entre estes Conceitos. Revista de Gestão e Projetos - GeP.

PMI. (2017). A guide to the project management body of knowledge (PMBOK guide). In Project Management Journal (Vol. 40).

Yin, R.K. Planejamento e Métodos, Bookman, (2a ed.), 2003.

\section{Apêndice}

\section{Questionário de Pesquisa}

1) Como foram identificados os stakeholders do projeto?

2) Houve um levantamento formal de requisitos envolvendo todos os stakeholders do projeto?

3) Quais os critérios adotados para classificar os stakeholders?

4) No planejamento da comunicação foram envolvidos todos os stakeholders?

5) Os stakeholders participaram do planejamento de riscos do projeto?

6) Foram identificadas mudanças significativas que impactassem o escopo e os stakeholders no projeto?

7) $\mathrm{O}$ gerente de projeto tinha experiência nesse tipo de projeto? O tema LGPDP era conhecido pelo gerente do projeto?

8) Quais as competências ou habilidades seriam necessárias ao gerente de projetos para evitar os problemas quanto a LGPDP?

9) Com base na pergunta anterior, quais as ações foram efetivadas? 